\title{
A Receiver Architecture For Dual-Functional Massive MIMO OFDM RadCom Systems
}

\author{
Murat Temiz ${ }^{1}$, Emad Alsusa ${ }^{2}$ and Laith Danoon ${ }^{3}$ \\ School of Electrical and Electronic Engineering, The University of Manchester, UK \\ $\left\{\right.$ murat.temiz $^{1}$, e.alsusa ${ }^{2}$, l.danoon $\left.{ }^{3}\right\} @$ manchester.ac.uk
}

\begin{abstract}
This study ${ }^{1}$ introduces a receiver architecture for dual-functional communication and radar (RadCom) base-stations (BS), which exploits the spatial diversity between the received radar and communication signals, and performs interference cancellation (IC) to successfully separate these signals. In the RadCom system under consideration, both communication and radar systems employ orthogonal frequency-division multiplexing (OFDM) waveforms with overlapping subcarriers. Employing OFDM waveform allows the BS to simultaneously perform uplink channel estimation on the narrow-band subcarriers to efficiently obtain full channel state information (CSI) between the users (UEs) and the BS antenna elements. The estimated CSI matrix is then utilized to acquire uplink data streams from the UEs by suppressing the inter-user interference and radar signals which arrive at the BS through unknown channels. After acquiring the UEs' data, radar signals are extracted from the received complex baseband signals by performing interference cancellation. The proposed method has been analyzed mathematically and verified by simulations under various conditions including CSI mismatch and high radar interference. The results show that 16QAM modulated uplink is outstandingly robust against radar interference and that having a large number of antennas significantly improves the performance of both communication and radar subsystems, cooperatively. This study shows that it is possible to distinguish radar and communication signals by employing large-scale antenna arrays to successfully realize a RadCom receiver for future communication networks.
\end{abstract}

Keywords: Massive MIMO, MIMO OFDM Radar, Successive Interference Cancellation, RadCom Receiver

\section{Introduction}

The growing number of radar sensing applications and increasing bandwidth demand of communication devices are expected to impose on these two systems to co-exist and share the same frequency spectrum since the available frequency resources are limited [1]. Accordingly, these two systems must be elaborately designed to avoid interfering significantly with each other and to improve their immunity against interference. This shared spectrum usage can be dynamically managed by coordinating frequency, time and spatial resources occupied by these systems, if some knowledge is shared between them [2]. Furthermore, more sophisticated solutions for this radar and communication convergence problem have been proposed including designing robust radar waveforms against interference [3], minimizing the communication power output by exploiting multi-user interference to avoid interfering with the radar system [4] or forcing the radar to beamform waveforms into null spaces of the communication interference channel [5]. On the other hand, some applications may need to cooperatively perform both sensing and communication operations. For example, in autonomous vehicle driving scenarios, the vehicles

\footnotetext{
${ }^{1}$ This study has been accepted to be published at IEEE ICC Workshop on Communication and Radar Spectrum Sharing 2020 This is a preprint version, not the final version of the manuscript which will be published at IEEE Xplore after the conference.

C) 2020 IEEE. Personal use of this material is permitted. Permission from IEEE must be obtained for all other uses, in any current or future media, including reprinting/republishing this material for advertising or promotional purposes, creating new collective works, for resale or redistribution to servers or lists, or reuse of any copyrighted component of this work in other works.
} 


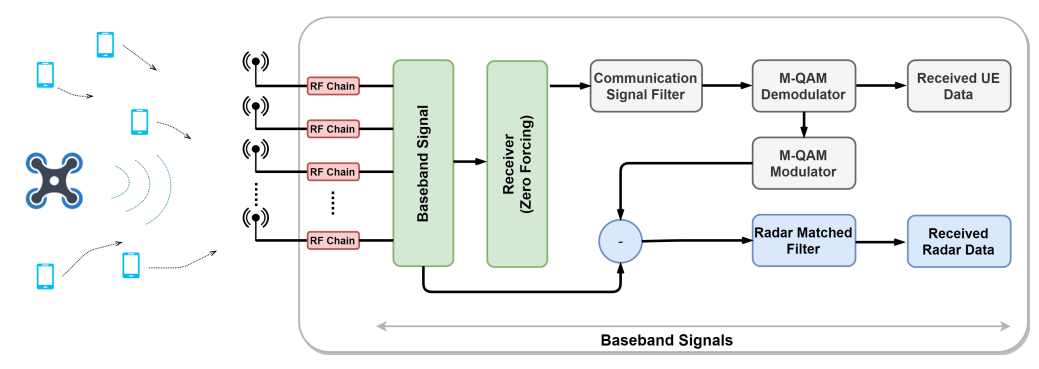

Figure 1: The proposed receiver architecture for a Massive MIMO RadCom system.

need to continuously observe the situation of the road and simultaneously communicate with other vehicles being present in the vicinity to enhance the safety of the autonomous driving. As a solution to this, communication and sensing can be jointly performed with the same frequency resources by a dual-functional radar and communication (RadCom) hardware to reduce cost as well as to provide enhanced spectrum and energy efficiencies. Several sophisticated methods have been proposed to effectively realize these RadCom systems while aiming to achieve satisfactory communication data rates and radar target estimation performance [6-9]. Shi et al. proposed a design strategy for an integrated OFDM radar waveform which also carries data to the downlink communication UEs for single antenna systems [6]. On the other hand, having large-scale antenna arrays may allow the BS to beamform data to the UEs and radar waveforms to the targets as shown in [7]. Furthermore, directional and omnidirectional radar waveform designs have also been considered to effectively utilize the entire antenna array for an integrated RadCom system [8]. Another approach proposes an antenna-selection based signaling method for RadCom systems to integrate communication symbols in radar waveforms [9] These aforementioned studies mainly focus on the transmitter part of the RadCom systems where communicating with downlink UEs and transmitting radar waveforms are jointly performed. For the receiver side, an interference cancellation method is proposed for the RadCom receivers in [10] where different OFDM subcarriers are initially allocated to radar and communication systems, and the interference caused by the subcarrier misalignment is alleviated using an interference cancellation method. Another study considers the self-interference between radar transmitter and receiver in full-duplex OFDM radars and applies analog and digital interference cancellation methods to improve radar detection performance [11]. Differently from the studies presented above, in this study, we propose a massive MIMO receiver architecture which can simultaneously acquire the symbols transmitted by multiple UEs and detect the radar returns reflected by the targets. The proposed technique exploits the estimated CSI of the UEs to suppress radar returns and inter-user interference to acquire their data, subsequently, reconstructs radar returns by subtracting these acquired symbols from the received baseband signals. This paper analytically examines the proposed technique and verifies its performance by QAM simulations, and shows that it can successfully separate the uplink communication data and the radar returns.

Notation Throughout the paper, matrices are denoted by bold uppercase letters (e.g. $\mathbf{H}$ ) and vectors are denoted by bold lowercase letters (e.g. g). Transpose and Hermitian transpose are denoted by [.] $]^{T}$ and [.] ${ }^{H}$, respectively. $\mathbb{E}[$.$] and \|$.$\| denote expected value and l_{2}$ norm operations, respectively.

\section{System Model}

This study focuses on successfully distinguishing uplink communication signals transmitted by the UEs and the radar returns from the targets. In the massive MIMO RadCom system under consideration, the BS is equipped with $M$ antennas simultaneously communicating with $K$ single-antenna UEs while detecting $U$ targets in the line-of-sight (LOS) of the BS. The UEs are assumed to be randomly located in the non-line-of-sight (NLOS) of the BS in the cell, resulting in flat Rayleigh fading channels. The proposed receiver architecture is illustrated in Fig 1 where the received signals consisting of radar returns and communication signals are, firstly, converted into baseband by the RF chain. Then, the communication signals are acquired by the zero-forcing (ZF) receiver which endeavors to cancel out inter-user interference and radar returns which arrive at the BS through unknown channels. Subsequently, the successfully detected communication symbols are multiplied by 


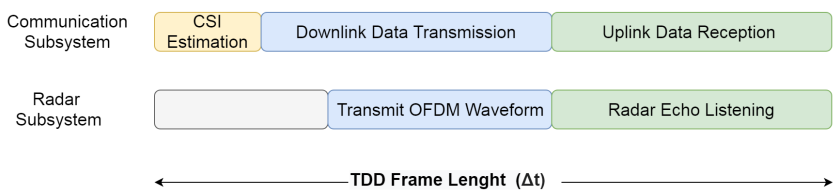

Figure 2: Synchronized TDD frame structure of the RadCom system.

the estimated CSI and then subtracted from the received baseband signal to obtain the radar returns. Timedivision-duplex (TDD) operation mode is considered since it requires only uplink CSI estimation. The duration of the TDD frame $\Delta t$ is smaller than the coherence time $\tau$, hence, the channel is assumed to be constant during one TDD frame. The operations of radar and communication subsystems are synchronized such that during the communication downlink transmission, the radar subsystem transmits OFDM radar waveform and during uplink, both communication signals and radar returns are simultaneously received as shown in Fig. 2. To precisely acquire the CSI of the UEs, the radar subsystem does not transmit any signal during the CSI estimation phase.

\subsection{Uplink OFDM Massive MIMO RadCom System Model}

The BS antenna array elements simultaneously receive the uplink signals transmitted by the UEs and the radar returns reflected by the targets being present in the propagation environment. It is worth noting that the radar clutter is omitted here as it is highly dependent on the environment and can be modeled as an additive noise. Accordingly, the equivalent baseband signal received by $M$ antenna elements of the BS, $\mathbf{y}(t)=$ $\left[\begin{array}{llll}y_{1}(t) & y_{2}(t) & \cdots & y_{M}(t)\end{array}\right]^{T}$, in each subcarrier can be given by

$$
\mathbf{y}(t)=\sqrt{\gamma} \mathbf{H x}(t)+\sqrt{\rho} \mathbf{g z}(t)+\mathbf{n}(t),
$$

where the average communication signal power transmitted by the UEs and the radar output power are denoted by $\gamma$ and $\rho$, respectively. $\mathbf{n}(t)=\left[\begin{array}{llll}n_{1}(t) & n_{2}(t) & \cdots & n_{M}(t)\end{array}\right]^{T}$ denotes the noise (AWGN) vector, where $n_{m} \sim \mathcal{C N}\left(0, \sigma_{n}^{2}\right)$ with zero mean and $\sigma_{n}^{2}$ noise variance. The channel matrix $\mathbf{H} \in \mathbb{C}^{M \times K}$ comprises complex channel coefficients between $K$ UEs and $M$ BS antenna elements. The channel vector between the $k$ th UE and $M$ BS antenna element is modeled as $\mathbf{h}_{k}=\beta_{k} \mathbf{f}_{k}$ where $\beta_{k}$ is the path-loss and log-normal shadowing of the $k$ th UE, and vector $\mathbf{f}_{k} \in \mathbb{C}^{M \times 1}$ contains the small-scale fading coefficients between the $k$ th UE and $M$ antenna elements as $f_{m, k} \sim \mathcal{C N}(0,1)$. The transmitted communication and radar signals have average unit power as $\mathbb{E}\left[\left|x_{k}\right|^{2}\right]=1$ and $\mathbb{E}\left[|z|^{2}\right]=1$. The transmitted OFDM radar waveform vector $\mathbf{z}(t)$, which contains $L$ QAM symbols filtered by a rectangular filter $($ rect (.)) in the $n$th subcarrier, is given by

$$
\mathbf{z}(t)=\sum_{\mu=1}^{\mu=L} d(\mu) e^{j 2 \pi f_{n} t} r e c t\left(\frac{t-\mu T}{T}\right),
$$

where $\mu$ denotes the index of the QAM modulated symbol $d$ with duration $T$ [12] and frequency of the subcarrier is denoted by $f_{n}=n \Delta f$, where $\Delta f$ denotes the subcarrier spacing. The radar returns received by the $m$ th antenna from the $u$ th target can be given by

$$
\mathbf{r}_{m, u}(t)=\sqrt{\rho} \mathbf{z}(t) a_{m, u}(t) e^{j 2 \pi f_{n}\left(t-\frac{2 R_{m, u}}{c_{o}}\right)} e^{j 2 \pi f_{D_{u}} t},
$$

where $a_{m, u}(t)$ denotes the two-way channel attenuation between $m$ th antenna and $u$ th target, given by

$$
a_{m, u}(t)=\sqrt{\frac{G_{T x} G_{R x} c^{2} \sigma_{R C S_{u}}}{(4 \pi)^{3} f_{c}^{2} R_{m, u}^{4}}},
$$

according to the well-known radar equation. $\sigma_{R C S_{u}}$ denotes the radar cross-section of the target, $f_{c}$ denotes the carrier frequency, $G_{T x}$ and $G_{R x}$ denote the transmit and receive antenna gains, respectively. The phase shift caused by the range of the target $R_{m, u}$ is given by $e^{j 2 \pi f_{n}\left(t-\frac{2 R_{m, u}}{c_{o}}\right)}$ and $f_{D_{u}}$ denotes the Doppler frequency 
shift due to the velocity of the $u$ th target. For a two-way radar propagation, the Doppler shift is given by $f_{D_{u}}=\frac{2 v_{u} f_{c}}{c}$ with regard to the velocity of the target $v_{u}$. The subcarrier spacing of the OFDM radar waveform is to be selected subject to $\Delta f>f_{D, \max }$ to ensure that orthogonality of the subcarriers is preserved after the Doppler shift [12]. Accordingly, the channel coefficient between the $m$ th antenna element and the $u$ th target can be found by (element-wise) dividing the received radar signal vector by the transmitted signal vector in the frequency domain as given by

$$
g_{m, u}(t)=\frac{\mathbf{r}_{m, u}(t)}{\sqrt{\rho} \mathbf{z}(t)}=a_{m, u}(t) e^{j 2 \pi f_{n}\left(t-\frac{2 R_{m, u}}{c_{o}}\right)} e^{j 2 \pi f_{D_{u}} t} .
$$

Let $g_{m}$ denote the channel between the $m$ th antenna and all targets i.e. $g_{m}=\sum_{u=1}^{U} g_{m, u}$. Vector $\mathbf{g} \in \mathbb{C}^{M \times 1}$ consists of channel coefficients between $M$ antennas and $U$ targets as $\mathbf{g}=\left[\begin{array}{llll}g_{1} & g_{2} & \cdots & g_{m}\end{array}\right]$. The channel vector $\mathbf{g}$ contains information about the location and velocity of the target, hence, it needs to be acquired precisely to identify these features. Therefore, the radar subsystem endeavors to recover the vector $\mathbf{g}$ to identify the objects while the main aim of the communication subsystem is to recover the data vector $\mathbf{x}$ transmitted by the UEs.

The communication channel $\mathbf{H}$ is estimated by the BS through uplink channel estimation, and the transmitted radar waveform vector $\mathbf{z}$ is also known since it is transmitted by the BS during the downlink. To suppress the inter-user interference and radar interference, the Zero-Forcing (ZF) receiver given by $\mathbf{W}=\hat{\mathbf{H}}\left(\hat{\mathbf{H}}^{H} \hat{\mathbf{H}}\right)^{-1}$ based on estimated communication channel matrix $\hat{\mathbf{H}}$ is employed. The received signal after $\mathrm{ZF}$ is given by

$$
\hat{\mathbf{x}}=\mathbf{W}^{H} \mathbf{y}=\mathbf{W}^{H} \sqrt{\gamma} \mathbf{H} \mathbf{x}+\mathbf{W}^{H} \sqrt{\rho} \mathbf{g z}+\mathbf{W}^{H} \mathbf{n},
$$

where the radar interference is expected to be surpassed by the receiver since radar signals are received through line-of-sight channels that have significantly low correlation with the communication channels which are mostly non-line-of-sight channels [13]. After that, the radar echos can be reconstructed by subtracting the detected communication symbols from the received baseband signal as

$$
\begin{aligned}
\mathbf{r}_{\text {rad }} & =\mathbf{y}-\sqrt{\gamma \hat{\mathbf{H}} \ddot{\mathbf{x}}} \\
& =[\sqrt{\gamma} \mathbf{H} \mathbf{x}-\sqrt{\gamma} \hat{\mathbf{H}} \ddot{\mathbf{x}}]+\sqrt{\rho} \mathbf{g z}+\mathbf{n}
\end{aligned}
$$

where $\ddot{\mathbf{x}}$ denotes the re-modulated symbols acquired by demodulation of $\hat{\mathbf{x}}$. Subsequently, the estimated channel of the targets is calculated by

$$
\begin{aligned}
\hat{\mathbf{g}} & =\frac{\mathbf{r}_{r a d}}{\sqrt{\rho} \mathbf{z}}=\frac{\sqrt{\gamma}[\mathbf{H} \mathbf{x}-\hat{\mathbf{H}} \ddot{\mathbf{x}}]+\sqrt{\rho} \mathbf{g z}+\mathbf{n}}{\sqrt{\rho} \mathbf{z}} \\
& =\mathbf{g}+\frac{\sqrt{\gamma}[\mathbf{H} \mathbf{x}-\hat{\mathbf{H}} \ddot{\mathbf{x}}]+\mathbf{n}}{\sqrt{\rho} \mathbf{z}}
\end{aligned}
$$

where the second term denotes the residual communication signals after IC and AWGN noise. This equation inherently indicates that the residual communication signals can be proliferated by the CSI mismatch or signal detection errors.

\section{Uplink Communication Capacity}

In this section, the achievable capacity of the uplink with radar interference is analyzed. The average signal-tonoise ratio (SNR) per user per antenna is defined as $\alpha=\gamma \beta_{k} / \sigma_{n}^{2}$. While emitting more powerful radar waveform may provide a better target detection accuracy, it can also cause more interference on the communication signals. Therefore, a power ratio between the average received radar and received communication powers by the BS array is defined as $\Upsilon=\frac{\gamma \beta_{k}}{\rho U a_{m}^{2}}$ to examine the relation between their power, communication data rate and radar detection performance, where $a_{m}$ denotes the two-way channel attenuation between the $m$ th antenna 
and the targets i.e. $a_{m}=\sum_{u=1}^{U} a_{m, u}$. The CSI estimation may be degraded by various factors such as noise, interference on the pilot symbols or RF mismatch between transmitter and receiver [14], hence, a generic CSI mismatch model has been employed. The BS is assumed to estimate the CSI with an error matrix as given by $\hat{\mathbf{H}}=\sqrt{1-\xi} \mathbf{H}+\mathbf{E}$ by utilizing uplink pilot symbols [15]. The complex valued channel estimation error matrix, $\mathbf{E} \in \mathbb{C}^{M \times K}$, consists of independent random values given by $e_{m k} \sim \mathcal{C N}(0, \xi)$ with 0 mean and variance $\xi=\eta \alpha^{-\beta}$, where the SNR is denoted by $\alpha$, and error coefficients $\eta$ and $\beta$ are defined to produce various CSI mismatch scenarios [16]. The acquired signal of the $k$ th UE at the BS can be given by

$$
\begin{aligned}
\hat{x}_{k}=\sqrt{\gamma} \mathbf{w}_{k}^{H} \mathbf{h}_{k} x_{k}+ \\
\sum_{i=1, i \neq k}^{K} \sqrt{\gamma} \mathbf{w}_{k}^{H} \mathbf{h}_{i} x_{i}+\sqrt{\rho} \mathbf{w}_{k}^{H} \mathbf{g z}+\mathbf{w}_{k}^{H} n,
\end{aligned}
$$

where $\mathbf{w}_{k}^{H} \mathbf{g z}$ corresponds to interfering radar signals with this UE's signals. The channel vector of the $i$ th UE can be rewritten as $\mathbf{h}_{i}=\frac{\hat{\mathbf{h}}_{i}-\mathbf{e}_{i}}{\sqrt{1-\xi}}$ according to the error model that has been previously explained. Substituting this error model in (9) yields

$$
\begin{aligned}
\hat{x}_{k} & =\sqrt{\gamma} \frac{1}{\sqrt{1-\xi}}\left(\mathbf{w}_{k}^{H} \hat{\mathbf{h}}_{k} x_{k}-\mathbf{w}_{k}^{H} \mathbf{e}_{k} x_{k}\right) \\
& +\sqrt{\gamma} \frac{1}{\sqrt{1-\xi}} \sum_{i=1, i \neq k}^{K}\left(\mathbf{w}_{k}^{H} \hat{\mathbf{h}}_{i} x_{i}-\mathbf{w}_{k}^{H} \mathbf{e}_{i} x_{i}\right) \\
& +\sqrt{\rho} \mathbf{w}_{k}^{H} \mathbf{g z}+\mathbf{w}_{k}^{H} n .
\end{aligned}
$$

When $M>>K, \mathbb{E}\left[\left|\mathbf{w}_{k}^{H} \hat{\mathbf{h}}_{k}\right|^{2}\right]=1$ and $\mathbb{E}\left[\left|\mathbf{w}_{k}^{H} \hat{\mathbf{h}}_{i}\right|^{2}\right]=0$ can be given [17]. Consequently, the average received useful signal power from the $k$ th $\mathrm{UE}$ is given

$$
\begin{aligned}
p_{k} & =\gamma \frac{1}{1-\xi} \mathbb{E}\left[\left|\mathbf{w}_{k}^{H} \hat{\mathbf{h}}_{k}-\mathbf{w}_{k}^{H} \mathbf{e}_{i}\right|^{2}\right] \\
& \approx \gamma\left(1-\xi+\frac{\xi}{1-\xi}\right),
\end{aligned}
$$

when $\xi<0.2$. The power of inter-user interference due to channel estimation errors is given by

$$
\begin{aligned}
p_{i} & =\gamma \frac{1}{1-\xi} \sum_{i=1, i \neq k}^{K} \mathbb{E}\left[\left|\mathbf{w}_{k}^{H} \hat{\mathbf{h}}_{i}-\mathbf{w}_{k}^{H} \mathbf{e}_{i}\right|^{2}\right] \\
& =\gamma \frac{K-1}{1-\xi} \mathbb{E}\left[\left|\mathbf{w}_{k}^{H} \mathbf{e}_{i}\right|^{2}\right] \approx \frac{\gamma(K-1) \xi}{(1-\xi)(M-K) \beta_{k}},
\end{aligned}
$$

and the effective noise after the ZF is given by

$$
p_{n}=\mathbb{E}\left[\left|\mathbf{w}_{k}^{H} \mathbf{n}\right|^{2}\right]=\mathbb{E}\left[\left\|\mathbf{w}_{k}^{H}\right\|^{2}\right] \sigma_{n}^{2}=\frac{\sigma_{n}^{2}}{(M-K) \beta_{k}} .
$$

Lastly, the power of the interfering radar signals is approximately given by

$$
\begin{aligned}
p_{r i} & =\rho \mathbb{E}\left[\left|\mathbf{w}_{k}^{H} \mathbf{g}\right|^{2}\right]=\rho \mathbb{E}\left[\left\|\mathbf{w}_{k}^{H}\right\|^{2}\right] \mathbb{E}\left[\left|g_{m}\right|^{2}\right] \\
& \approx \frac{\rho U a_{m}^{2}}{(M-K) \beta_{k}},
\end{aligned}
$$

since $\mathbf{w}_{k}$ and $\mathbf{g}$ are uncorrelated and independent, $\mathbb{E}\left[\left\|\mathbf{w}_{k}^{H}\right\|^{2}\right]=\frac{1}{(M-K) \beta_{k}}[17]$ and $\mathbb{E}\left[\left|g_{m}\right|^{2}\right]=U a_{m}^{2}$ for $U$ targets with average path-loss $a_{m}$ between the $m$ th antenna and the targets. Note that the details of the proofs are omitted here due to the limited space. Finally, the SINR for the $k$ th UE is given by 


$$
S I N R_{k} \approx \frac{\gamma\left(1-\xi+\frac{\xi}{1-\xi}\right)(M-K) \beta_{k}}{\frac{\gamma \xi(K-1)}{1-\xi}+\sigma_{n}^{2}+\rho U a_{m}^{2}} .
$$

The channel capacity of the $k$ th UE is given by $C_{k}=\log _{2}\left(1+S I N R_{k}\right)$. If there is no radar interference on the communication signals i.e. $\rho=0$ with perfect CSI knowledge i.e. $\xi=0$, then the capacity of the $k$ th user in the RadCom network approaches to $C_{k}=\log _{2}\left(1+\frac{\gamma(M-K) \beta_{k}}{\sigma_{n}^{2}}\right)$ which is considered as the lower-bound on the capacity of the ZF receiver within favorable channel conditions [17]. The radar interference term can be reduced by having more BS antennas according to (14) because more BS antennas will increase the spatial resolution of the array, resulting in a better interference suppression performance.

\section{Radar Channel Estimation Performance}

OFDM radars exploit the estimated channel information between the radar and targets to identify the velocity, range and the relative position of the targets [12]. For this reason, the accuracy of the target channel estimation determines the radar estimation performance. On this account, the normalized mean squared error (NMSE), $\Phi_{g}$, between the estimated radar channel $\hat{\mathbf{g}}$ and the real radar channel $\mathbf{g}$ is used as a performance metric here. The average NMSE of the radar channel estimation over $I$ iteration is given by

$$
\Phi_{g}=\frac{\mathbb{E}\left[\left\|\left(\mathbf{g}_{i}-\hat{\mathbf{g}}_{i}\right)\right\|^{2}\right]}{\mathbb{E}\left[\left\|\mathbf{g}_{i}\right\|^{2}\right]} .
$$

where subscript $i=[1,2,, I]$ denotes the iteration index of the simulations. Substituting the estimated radar channel matrix given by (8) in (16) yields

$$
\Phi_{g}=\mathbb{E}\left[\left\|\left(\frac{\sqrt{\gamma}(\mathbf{H x}-\hat{\mathbf{H}} \ddot{\mathbf{x}})+\mathbf{n}}{\sqrt{\rho} \mathbf{z}}\right)_{i}\right\|^{2}\right] \frac{1}{\mathbb{E}\left[\left\|\mathbf{g}_{i}\right\|^{2}\right]},
$$

which intuitively indicates that the residual communication signals and noise after IC can degrade the radar estimation performance while having more radar transmit power $(\rho)$ can improve the accuracy of the radar estimation. However, having more radar power will also degrade the communication signal detection by causing more interference as stated by (14), and this also may cause more residual communication signals interfering with radar after IC. If the CSI and symbols are detected flawlessly, then $\hat{\mathbf{H}}=\mathbf{H}$ and $\hat{\mathbf{x}} \approx \ddot{\mathbf{x}}=\mathbf{x}$ will be obtained, and this corresponds to that all communication interference on the radar can be eliminated as $\sqrt{\gamma}(\mathbf{H} \mathbf{x}-\hat{\mathbf{H}} \hat{\mathbf{x}})=0$, hence, the best possible radar channel detection performance can be given by

$$
\Phi_{g}=\frac{\mathbb{E}\left[\left\|\mathbf{n}_{i}\right\|^{2}\right]}{\rho \mathbb{E}\left[\left\|\mathbf{g}_{i}\right\|^{2}\right]} .
$$

The uplink capacity and radar estimation performance analyses reveal that the performance of both systems may be systematically improved by having more antennas, implementing superior IC algorithms or estimating more accurate communication CSI.

\section{Simulations and Numerical Results}

To verify the capacity analysis presented in section 3, Monte-Carlo simulations of the RadCom system has been performed. In the simulations, a fixed number of UEs $(K=10)$ simultaneously transmit randomly generated modulated data through the communication channel to the BS while it receives radar returns from the targets. Three different modulation schemes, namely 16QAM, 32QAM and 64QAM, are considered for the modulation of communication data and OFDM radar waveform. The received baseband signals are processed by the proposed method presented in Fig. 1 to acquire messages from the UEs and channel information of the single target. The general parameters used in the simulations are given in Table 1.

The power-ratio $\Upsilon$ between the received radar and communication signals is set to $0,0.5$ and 1 to test different radar interference situations. $\Upsilon=0$ corresponds to that the radar does not operate during that period, 
Table 1: The parameters are used in the simulations.

\begin{tabular}{|c|c|c|}
\hline Parameter & Explanation & Value \\
\hline \hline$f_{c}$ & Carrier frequency & $5 \mathrm{GHz}$ \\
\hline$R$ & Target Range & Random $[50-200 \mathrm{~m}]$ \\
\hline$\sigma_{R C S}$ & Target RCS & $0.4 \mathrm{~m}^{2}$ \\
\hline$\beta, \eta$ & CSI error parameters & $0.8,0.5$ \\
\hline$K$ & Number of UEs & 10 \\
\hline$U$ & Number of targets & 1 \\
\hline$M$ & Number of antennas & 20 to 200 \\
\hline
\end{tabular}

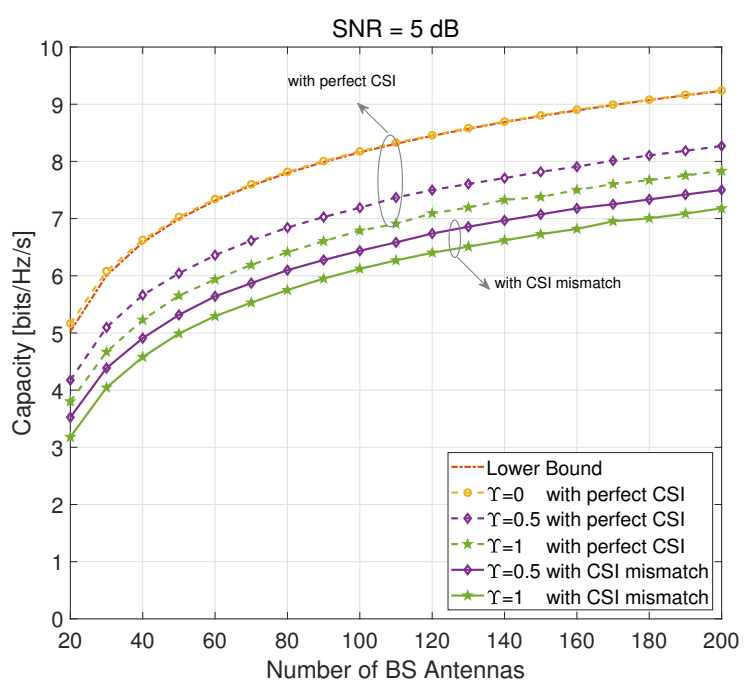

Figure 3: Capacity of uplink RadCom system as a function of number of BS antennas. $S N R=5 \mathrm{~dB}, K=10$ $\Upsilon=\{0,0.5,1\}$.

hence, only the communication signals are received. The capacity of the uplink under radar interference is analyzed using (15) with various number of antennas. Fig. 3 illustrates this capacity analysis at $5 \mathrm{~dB}$ SNR with $\Upsilon=\{0,0.5,1\}$. Lower bound on the capacity of the ZF is also illustrated as a reference in this figure. This figure reveals that when the received powers from the communication and radar systems are equal, the capacity is dropped by only $1.4 \mathrm{bits} / \mathrm{Hz} / \mathrm{s}$ compared to the case where there is no radar interference. CSI mismatch further degraded the capacity as it is expected, and this capacity drop caused by CSI mismatch is nearly constant with different number of antennas. Lower bound of the ZF capacity is reached when the radar interference does not exist, i.e. $\Upsilon=0$, as expected. The capacity increases as the number of antennas grows, however, the gap between the capacities with and without radar interference is nearly fixed. Fig. 4 illustrates the performance of 16QAM, 32QAM and 64QAM schemes without channel coding and with 100 antennas with the proposed technique. It shows that 16QAM is outstandingly robust against the radar interference due to relatively large distances between its constellation points. It can reach its capacity when the SNR is sufficient even with $\rho=\gamma$ i.e. $\Upsilon=1$. 32QAM and 64QAM modulated uplinks can also handle the radar interference when $\Upsilon=0.5$. However, especially 64QAM uplink suffers from high radar interference (i.e. when $\Upsilon=1$ ) and its capacity is degraded by around $0.7 \mathrm{bits} / \mathrm{Hz} / \mathrm{s}$ per user.

On the other hand, radar target estimation accuracy is another important metric for an OFDM RadCom receiver. While detecting QAM modulated communication symbols as shown in Fig 4, the radar returns from the target are acquired by the proposed IC technique. Two different methods are considered, which are canceling communication signals just after the ZF receiver by subtracting it from the received signals and the other one includes one extra stage which is demodulation and re-modulation of the signals as shown in Fig. 1. This extra stage corrects the constellation diagram if the QAM symbols are detected flawlessly, otherwise, it may also 


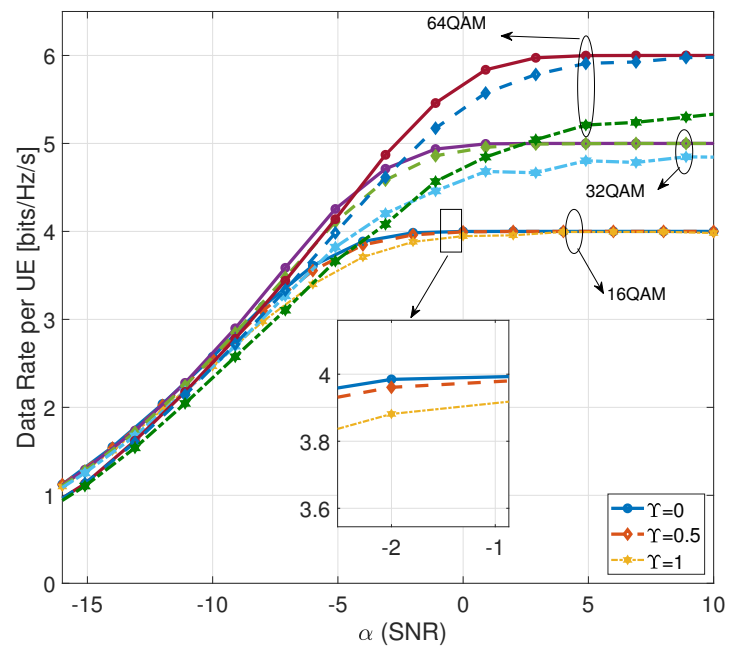

Figure 4: Uplink data-rate with 16QAM, 32QAM and 64QAM under radar interference with perfect CSI. $M=100, K=10$

amplify the detection errors. Fig. 5 illustrates the NMSE of the radar channel detection $\Phi_{g}$ per BS antenna with 16QAM and 64QAM modulations. The first two legends indicate that the $\Phi_{g}$ obtained using only $\hat{\mathbf{x}}$ which is the straightforward output of the ZF. This method exhibited lower errors at low SNRs, however, it suffers from an error floor which is between $\Phi_{g}=0.07$ and $\Phi_{g}=0.09$ at high SNRs. Other lines indicate the NMSE of obtained radar channels using $\ddot{\mathbf{x}}$ which is the re-modulation of $\hat{\mathbf{x}}$. These have much higher errors at low SNRs, which are mainly caused by the detection errors of the QAM signals. However, these errors are alleviated by the increased SNR and the target channel information is estimated with lower errors at high SNRs and this method does present any error floor. Having more radar power has significantly enhanced the detection accuracy with 16QAM. However, high radar power output (i.e. $\Upsilon=1$ ) has caused lower target detection performance with 64QAM, this is mainly due to the detection errors of 64QAM caused by the high radar interference which also can be seen in Fig. 4. This section verifies that communication and radar performances are associated with each other and can be mutually improved by implementing adequate IC methods or having more antennas.

\section{Conclusion}

A receiver architecture for massive MIMO OFDM RadCom has been proposed, which utilizes channel diversity between the UEs and targets to successfully receive the data symbols transmitted by multiple UEs and acquire the radar returns simultaneously. Firstly, the radar returns are recognized as interference and zero forced to obtain the uplink symbols from the UEs. Then, the baseband communication signals are reconstructed by utilizing these obtained symbols and subtracted from the received signals to obtain the radar returns. The uplink capacity of the proposed receiver architecture has been analytically investigated under CSI mismatch and radar interference. In addition, the proposed architecture with 16QAM, 32QAM and 64QAM uplink are simulated to verify the analytical results. The results have shown that the communication symbols and the radar target channel information can be jointly estimated by employing large-scale antenna arrays. Especially with 16QAM, the proposed RadCom receiver may satisfactorily and efficiently operate due to the relatively large distances between its constellation points. As a future extension of this work, we will be focusing on enhancing the joint performance of the communication and radar subsystems by developing superior IC algorithms. 


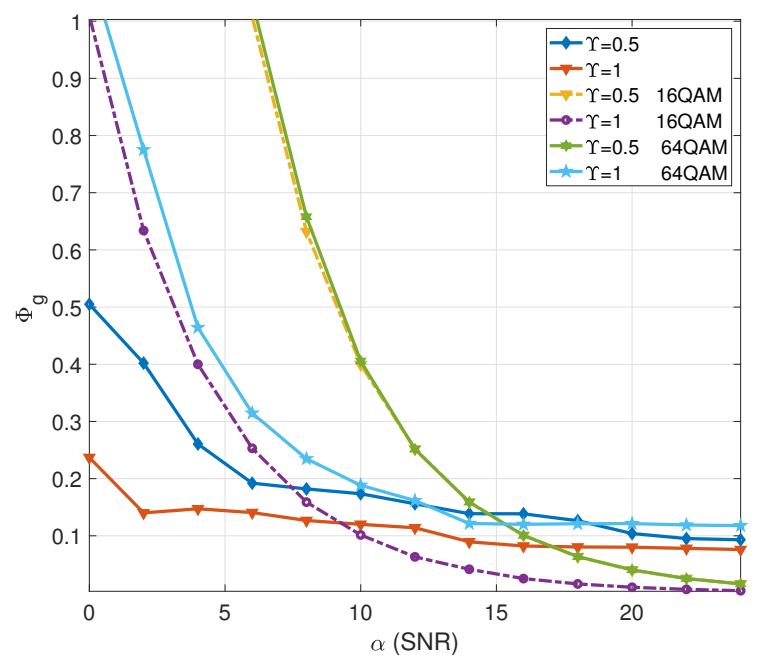

Figure 5: NMSE $\left(\Phi_{g}\right)$ of estimated radar channel information using reconstructed radar returns after IC.

\section{References}

[1] B. Paul, A. R. Chiriyath, and D. W. Bliss, "Survey of RF communications and sensing convergence research," IEEE Access, vol. 5, pp. 252-270, 2017.

[2] A. R. Chiriyath, B. Paul, and D. W. Bliss, "Radar-communications convergence: Coexistence, cooperation, and co-design," IEEE Transactions on Cognitive Communications and Networking, vol. 3, pp. 1-12, March 2017.

[3] C. Shi, F. Wang, M. Sellathurai, J. Zhou, and S. Salous, "Power minimization-based robust OFDM radar waveform design for radar and communication systems in coexistence," IEEE Transactions on Signal Processing, vol. 66, pp. 1316-1330, March 2018.

[4] F. Liu, C. Masouros, A. Li, T. Ratnarajah, and J. Zhou, "Mimo radar and cellular coexistence: A powerefficient approach enabled by interference exploitation," IEEE Transactions on Signal Processing, vol. 66, pp. 3681-3695, July 2018.

[5] S. Sodagari, A. Khawar, T. C. Clancy, and R. McGwier, "A projection based approach for radar and telecommunication systems coexistence," in 2012 IEEE Global Communications Conference (GLOBECOM), pp. 5010-5014, Dec 2012.

[6] C. Shi, F. Wang, S. Salous, and J. Zhou, "Low probability of intercept-based optimal OFDM waveform design strategy for an integrated radar and communications system," IEEE Access, vol. 6, pp. 57689-57699, 2018 .

[7] F. Liu, C. Masouros, A. Li, H. Sun, and L. Hanzo, "MU-MIMO communications with MIMO radar: From co-existence to joint transmission," IEEE Transactions on Wireless Communications, vol. 17, pp. 2755-2770, April 2018.

[8] F. Liu, L. Zhou, C. Masouros, A. Li, W. Luo, and A. Petropulu, "Toward dual-functional radarcommunication systems: Optimal waveform design," IEEE Transactions on Signal Processing, vol. 66, pp. 4264-4279, Aug 2018.

[9] X. Wang, A. Hassanien, and M. G. Amin, "Dual-function MIMO radar communications system design via sparse array optimization," IEEE Transactions on Aerospace and Electronic Systems, vol. 55, pp. 1213-1226, June 2019. 
[10] Y. L. Sit, B. Nuss, and T. Zwick, "On mutual interference cancellation in a MIMO OFDM multiuser radar-communication network," IEEE Transactions on Vehicular Technology, vol. 67, pp. 3339-3348, April 2018.

[11] C. Baquero Barneto, T. Riihonen, M. Turunen, L. Anttila, M. Fleischer, K. Stadius, J. Ryynanen, and M. Valkama, "Full-duplex OFDM radar with LTE and 5G NR waveforms: Challenges, solutions, and measurements," IEEE Transactions on Microwave Theory and Techniques, vol. 67, pp. 4042-4054, Oct 2019 .

[12] C. Sturm and W. Wiesbeck, "Waveform design and signal processing aspects for fusion of wireless communications and radar sensing," Proceedings of the IEEE, vol. 99, pp. 1236-1259, July 2011.

[13] M. Temiz, Y. Zhang, E. Alsusa, and L. Danoon, "Investigation of channel correlation in indoor wideband massive MIMO systems," in 2019 IEEE International Symposium on Antennas and Propagation and USNCURSI Radio Science Meeting, pp. 1577-1578, July 2019.

[14] M. Temiz, E. Alsusa, and L. Danoon, "Impact of imperfect channel estimation and antenna correlation on quantised massive multiple-input multiple-output systems," IET Communications, vol. 13, no. 9, pp. 1262$1270,2019$.

[15] D. Mi, M. Dianati, L. Zhang, S. Muhaidat, and R. Tafazolli, "Massive mimo performance with imperfect channel reciprocity and channel estimation error," IEEE Transactions on Communications, vol. 65, pp. 3734-3749, Sep. 2017.

[16] P. Aquilina and T. Ratnarajah, "Performance analysis of IA techniques in the MIMO IBC with imperfect csi," IEEE Transactions on Communications, vol. 63, pp. 1259-1270, April 2015.

[17] H. Q. Ngo, E. G. Larsson, and T. L. Marzetta, "Energy and spectral efficiency of very large multiuser mimo systems," IEEE Transactions on Communications, vol. 61, pp. 1436-1449, April 2013. 